\title{
DIFFERENTIAL FLATNESS THEORY-BASED CONTROL AND FILTERING FOR A MOBILE MANIPULATOR
}

\author{
Gerasimos G. Rigatos \\ Unit of Industrial Automation \\ Industrial Systems Institute \\ 26504, Rion Patras, Greece \\ grigat@ieee.org
}

Article history:

Received 15.09.2019, Accepted 10.12.2019

\begin{abstract}
The article proposes a differential flatness theorybased control and filtering method for the model of a mobile manipulator. This is a difficult control and robotics problem due to the system's strong nonlinearities and due to its underactuation. Using the Euler-Lagrange approach, the dynamic model of the mobile manipulator is obtained. This is proven to be a differentially flat one, thus confirming that it can be transformed into an input-output linearized form. Through a change of state and control inputs variables the dynamic model of the manipulator is finally written into the linear canonical (Brunovsky) form. For the latter representation of the system's dynamics the solution of both the control and filtering problems becomes possible. The global asymptotic stability properties of the control loop are proven. Moreover, a differential flatness theory-based state estimator, under the name of Derivative-free nonlinear Kalman Filter, is developed. This comprises (i) the standard Kalman Filter recursion on the linearized equivalent model of the mobile manipulator and (ii) an inverse transformation, relying on the differential flatness properties of the system which allows for estimating the state variables of the initial nonlinear model. Finally, by redesigning the aforementioned Kalman Filter as a disturbance observer one can achieve estimation and compensation of the disturbance inputs that affect the model of the mobile manipulator.
\end{abstract}

\section{Key words}

Mobile manipulators, differential flatness theory, flat outputs, canonical forms, global linearization, global stability, Kalman Filtering, disturbance observer.

\section{Introduction}

Mobile manipulators are widely used in several industrial and human assisting tasks. For instance they can be used in pick and placement tasks and for carrying objects, in assembling, in painting, spraying, harvesting, for patrolling and defence purposes, as well as for providing services to the elderly and the disabled $[\mathrm{Li}$ et al., 2009], [Dai and Liu, 2017], [Abeygunawardhana and Murakami, 2010], [Andaluz et al., 2015], [Li et al., 2008]. Dexterity and accuracy in the handling of objects as well as in the maneuvers performed by the mobile manipulators depend on the efficiency of the related control algorithms [Rigatos and Busawon, 2018], [Boyle et al., 2003], [Kocemarek et al., 2017], [Koraye and Nekao, 2016]. There are several results on nonlinear control approaches for robotic vehicles and mobile manipulators [Rigatos, 2011], [Rigatos, 2015], [Li et al., 2008], [Li et al., 2016], [Najjaran and Goldenberg, 2007]. In particular, the application of sliding-mode and backstepping methods can be hindered by the need to transform previously the dynamic model of mobile manipulators into canonical or triangular state-space forms. One can also note results on robust and adaptive control schemes for mobile manipulators which aim at compensating for model uncertainty and disturbances in these robotic systems [Xu et al., 2009], [Souzanchi et al., 2017], [Wu et al., 2014], [Park et al., 2018], [Monzur and Kulawik, 2006]. There are also findings on global linearizationbased control schemes for mobile manipulators, as for instance in the case of flatness-based control [Tang et al., 2011], [Morales et al., 2014], [Lévine, 2011], [Fliess and Mounier, 1999], [Sira-Ramirez and Agrawal, 2004], [Villagra et al., 2007]. Apart from motion control and the end-effector's positioning problem for mobile manipulators, compliance tasks and joint position and force control problems for the end-effector have been also analyzed [Galicki, 2016], [Linn and Goldenberg, 2002], [Mai and Wang, 2014], [Li et al., 2010], [Liu and Liu, 2009]. The development of functional mobile manipula- 
tors is completed with the solution of the related motion planning and trajectory generation problems.

In the present article, a differential flatness theorybased approach is developed for solving the control and state estimation problems in mobile manipulators under parametric model uncertainty and external disturbances [Rigatos and Busawon, 2018], [Rigatos, 2011], [Rigatos, 2015]. First the dynamic model of the mobile manipulator, comprising a four-wheel vehicle and a two-DOF robotic manipulator, is obtained through the application of the Euler-Lagrange analysis. It is proven that all state variables and the control inputs of the dynamic model can be written as differential functions of a subset of its state-vector elements, the so-called flat outputs of the system. Besides it is shown that the flat outputs of the system are differentially independent, meaning that both these variables and their derivatives are not connected through a relation in the form of a linear differential equation. These come to confirm that the dynamic model of the mobile manipulator is a differentially flat one. By proving the differential flatness of the mobile manipulator it is confirmed that (i) it can be transformed into an equivalent input-output linearized form, (ii) it can be written in the canonical (Brunovsky) state-space form.

For the linearized state-space representation of the robotic system, both the solution of its control and state estimation problem becomes possible. Actually, to solve the control problem one can apply pole-placement methods or optimal control approaches on the equivalent linearized description of the system. Moreover, to solve the associated state estimation problem a filtering method under the name of Derivative-free nonlinear Kalman Filter can be used [Rigatos and Tzafestas, 2007], [Basseville and Nikiforov, 1993], [Rigatos and Zhang, 2009]. This filtering approach consists of the standard Kalman Filter recursion on the equivalent linearized description of the system and of an inverse transformation that provides estimates for the state variables of the initial nonlinear model of the mobile manipulator. Moreover, by redesigning the aforementioned Kalman Filter as a disturbance observer, one can also estimate in real-time and compensate for additive input disturbances that affect the mobile manipulator. To this end, the state vector of the robotic system is extended by considering as additional state variables the disturbance inputs and their time derivatives.

The structure of the article is as follows: in Section 2 the dynamic model of the robotic manipulator is obtained after applying the Euler-Lagrange analysis. In Section 3 the differential flatness properties of the model of the mobile manipulator are proven. In Section 4 a flatness-based controller is designed for the mobile manipulator and estimation of its state variables is performed with the use of the Derivative-free nonlinear Kalman Filter. In Section 5 the aforementioned Kalman Filter is redesigned as a disturbance observer, thus allowing to estimate and compensate for the perturbations and model uncertainty terms of the mobile manipulator. In Section 6 the performance of the differential flatness theory-based control and estimation scheme is evaluated through simulation experiments. Finally, in Section 7 concluding remarks are given.

\section{Dynamic Model of the Mobile Manipulator}

To obtain the dynamic model of the mobile manipulator (Fig. 1) the Lagrangian functions of both the robotic manipulator and of the wheeled platform are computed first. The mass of the wheeled platform is denoted by $M$ and its moment of inertia for rotation around the vertical axis is denoted as $I_{z}$. The mass of the first link of the robotic manipulator is $m_{1}$ and the associated moment of inertia (for rotation around its center of gravity) is $I_{1}$. The mass of the second link of the robotic manipulator is $m_{2}$ and the associated moment of inertia (for rotation around its center of gravity) is $I_{2}$. The inertial reference frame of the system is denoted as $O_{1} X_{1} Y_{1} Z_{1}$ while the body-fixed reference frame is denoted as $O_{M} X_{M} Y_{M} Z_{M}$. The angle between the transversal axis of the vehicle and the $O X_{1}$ axis is denoted as $\psi$. The turn angle of the steering wheels of the vehicle with respect to its transversal axis is denoted as $\theta$. The turn angles of the joints of the robotic manipulator are denoted as $\theta_{1}$ and $\theta_{2}$ respectively.

Computation of the Lagrangian of the robotic manipulator: About link 1 it holds

$$
\begin{gathered}
K_{1}=\frac{1}{2} m_{1}\left(l_{c_{1}} \dot{\theta}_{1}\right)^{2}+\frac{1}{2} I_{1} \dot{\theta}_{1}^{2}+\frac{1}{2} m_{1}\left(V_{x}^{2}+V_{y}^{2}\right)+ \\
+\frac{1}{2} m_{1}\left(l_{c_{1}} \dot{\psi}\right)^{2}+\frac{1}{2} I_{1} \dot{\psi}^{2} \\
P_{1}=m_{1} g l_{c_{1}} \cos \left(\theta_{1}\right)
\end{gathered}
$$

About link 2 it holds

$$
\begin{gathered}
K_{2}=\frac{1}{2} m_{2} v_{c_{2}} v_{c_{2}}^{T}+\frac{1}{2} I_{2}\left(\dot{\theta}_{1}^{2}+\dot{\theta}_{2}^{2}\right)+ \\
+\frac{1}{2} m_{2}\left(V_{x}^{2}+V_{y}^{2}\right)+\frac{1}{2} m_{2}\left\{\left[l_{1}+l_{c_{2}} \cos \left(\theta_{2}\right)\right] \dot{\psi}\right\}^{2}+ \\
+\frac{1}{2} I_{2} \dot{\psi}^{2}
\end{gathered}
$$

where the velocity of its center of gravity is $v_{c_{2}}=$ $\left[\dot{x}_{c_{2}}, \dot{y}_{c_{2}}\right]$ and after intermediate operations one gets

$$
\begin{gathered}
v_{c_{2}} v_{c_{2}}^{T}=l_{1}^{2} \dot{\theta}_{1}^{2}+l_{c_{1}}^{2}\left(\dot{\theta}_{1}+\right. \\
\left.+\dot{\theta}_{2}\right)^{2}+2 l_{1} l_{c_{2}} \cos \left(\theta_{2}\right) \dot{\theta}_{1}\left(\dot{\theta}_{1}+\dot{\theta}_{2}\right)
\end{gathered}
$$

Thus the kinetic energy of the second link is given by

$$
\begin{gathered}
K_{2}=\frac{1}{2} m_{2}\left[l_{1}^{2} \dot{\theta}_{1}^{2}+l_{c_{1}}^{2}\left(\dot{\theta}_{1}+\right.\right. \\
\left.\left.+\dot{\theta}_{2}\right)^{2}+2 l_{1} l_{c_{2}} \cos \left(\theta_{2}\right) \dot{\theta}_{1}\left(\dot{\theta}_{1}+\dot{\theta}_{2}\right)\right]+ \\
+\frac{1}{2} I_{2}\left(\dot{\theta}_{1}^{2}+\dot{\theta}_{2}^{2}\right)+\frac{1}{2} m_{2}\left(V_{x}^{2}+V_{y}^{2}\right)+ \\
+\frac{1}{2} m_{2}\left\{\left[l_{1}+l_{c_{2}} \cos \left(\theta_{2}\right)\right] \dot{\psi}\right\}^{2}+\frac{1}{2} I_{2} \dot{\psi}^{2}
\end{gathered}
$$


It holds that

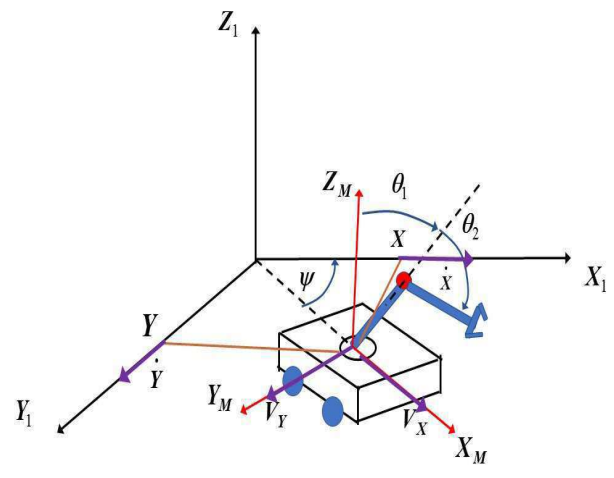

Figure 1. Diagram of the mobile manipulator together with the bodyfixed and the inertial reference frames

The potential energy of the second link of the manipulator is given by

$$
P_{2}=m_{2} g\left(l_{1} \cos \left(\theta_{1}\right)\right)+l_{c 2} \cos \left(\theta_{1}+\theta_{2}\right)
$$

The Lagrangian of the robotic manipulator is given by:

$$
L_{r}=K_{1}+K_{2}-P_{1}-P_{2}
$$

while the detailed description of the Lagrangian is

$$
\begin{gathered}
L_{r}=\frac{1}{2} m_{1}\left(l_{c_{1}} \dot{\theta}_{1}\right)^{2}+\frac{1}{2} I_{1} \dot{\theta}_{1}^{2}+ \\
+\frac{1}{2} m_{1}\left(V_{x}^{2}+V_{y}^{2}\right)+\frac{1}{2} m_{1}\left(l_{c_{1}} \dot{\psi}\right)^{2}+\frac{1}{2} I_{1} \dot{\psi}^{2} \\
\frac{1}{2} m_{2}\left[l_{1}^{2} \dot{\theta}_{1}^{2}+l_{c_{1}}^{2}\left(\dot{\theta}_{1}+\dot{\theta}_{2}\right)^{2}+\right. \\
\left.+2 l_{1} l_{c_{2}} \cos \left(\theta_{2}\right) \dot{\theta}_{1}\left(\dot{\theta}_{1}+\dot{\theta}_{2}\right)\right]+ \\
+\frac{1}{2} I_{2}\left(\dot{\theta}_{1}^{2}+\dot{\theta}_{2}^{2}\right)+\frac{1}{2} m_{2}\left(V_{x}^{2}+V_{y}^{2}\right)+ \\
+\frac{1}{2} m_{2}\left\{\left[l_{1}+l_{c_{2}} \cos \left(\theta_{2}\right)\right] \dot{\psi}\right\}^{2}+\frac{1}{2} I_{2} \dot{\psi}^{2} \\
-\left[m_{1} g l_{c_{1}} \cos \left(\theta_{1}\right)\right]-m_{2} g\left[\left(l_{1} \cos \left(\theta_{1}\right)\right)+l_{c 2} \cos \left(\theta_{1}+\theta_{2}\right)\right]
\end{gathered}
$$

Computation of the Lagrangian of the robotic vehicle: The kinetic energy of the vehicle is

$$
K_{v}=\frac{1}{2} M\left(V_{x}^{2}+V_{y}^{2}\right)+\frac{1}{2} I_{z} \dot{\psi}^{2}
$$

The vehicle's velocity is initially expressed in the body-fixed reference frame $O_{M} X_{M} Y_{M} Z_{M}$ (Fig. 1) and is given by the vector $\left[V_{x}, V_{y}\right]$. When the vehicle's velocity is written in the inertial reference frame $O_{1} X_{1} Y_{1} Z_{1}$, then it described by the vector $[\dot{X}, \dot{Y}]$. It holds that

$$
\begin{aligned}
V_{x} & =\cos (\psi) \dot{X}-\sin (\psi) \dot{Y} \\
V_{y} & =\sin (\psi) \dot{X}+\cos (\psi) \dot{Y}
\end{aligned}
$$

$$
V_{x}^{2}+V_{y}^{2}=\dot{X}^{2}+\dot{Y}^{2}
$$

Consequently, the kinetic energy of the vehicle is also written as

$$
K_{v}=\frac{1}{2} M\left(\dot{X}^{2}+\dot{Y}^{2}\right)+\frac{1}{2} I_{z} \dot{\psi}^{2}
$$

The potential energy of the vehicle is taken to be zero, considering that the vertical distance between its center of gravity and the ground is negligible.

$$
P_{v}=0
$$

Thus, about the Lagrangian of the vehicle one has:

$$
L_{v}=K_{v}-P_{v}
$$

and after using the previous relations the Lagrangian of the vehicle becomes

$$
L_{v}=\frac{1}{2} M\left(\dot{X}^{2}+\dot{Y}^{2}\right)+\frac{1}{2} I_{z} \dot{\psi}^{2}
$$

The aggregate Lagrangian of the mobile manipulator is

$$
L=L_{v}+L_{r}
$$

Next, the state vector for the mobile manipulator is defined as $x=\left[X, \dot{X}, Y, \dot{Y}, \psi, \dot{\psi}, \theta_{1}, \dot{\theta}_{1}, \theta_{2}, \dot{\theta}_{2}\right]^{T}$. The control inputs which are applied to the model of the mobile manipulator are as follows: (i) force $F$ which is generated by the vehicle's electric motor, (ii) Force $F_{b}$ which is generated by the vehicle's brakes, (ii) torques $T_{1}$ and $T_{2}$ which are generated by the actuators of the manipulator.

The force giving propulsion to the vehicle is $F$. It is considered that the vector of force $F$ forms an angle $\theta$ with the longitudinal axis of the vehicle (this is the angle of the steering wheels), and that the angle between this axis and the $O_{1} X_{1}$ axis of the inertial reference system is $\psi$ (Fig. 2).Moreover it is considered that a breaking force $F_{b}$ is exerted at the rear wheels of the vehicle, which is aligned with the longitudinal axis of the vehicle. Thus, one has that the aggregate force exerted on the vehicle along the $O_{1} X_{1}$ axis is $F_{X}=F \cos (\psi-\theta)-F_{b} \cos (\psi)$. Equivalently, the aggregate force that is exerted on the vehicle along the $O_{1} Y_{1}$ axis is $F_{Y}=F \sin (\psi-\theta)-F_{b} \sin (\psi)$. Moreover, considering that the distance between the front wheels 


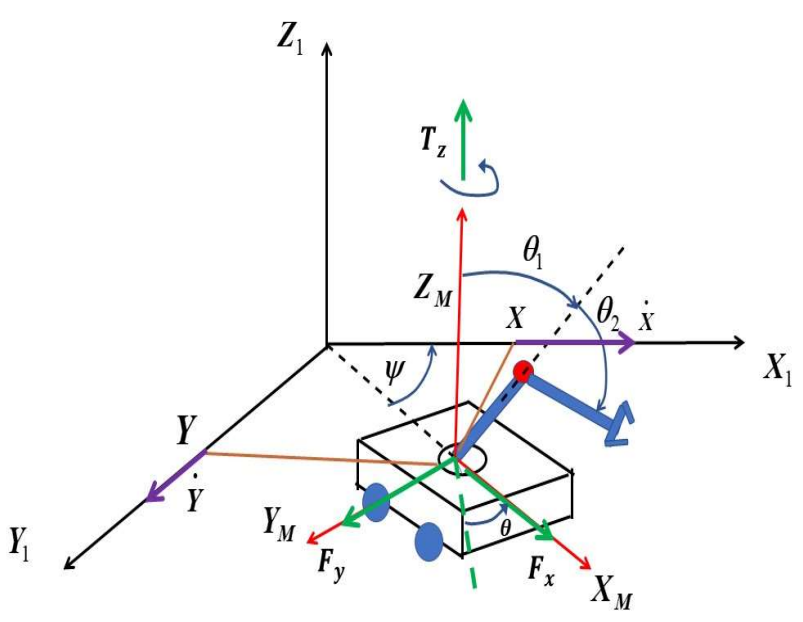

Figure 2. Diagram of the forces and torques exerted on the robotic vehicle

axis of the vehicle and its the transversal axis is $\bar{L}$, one has that the torque that causes rotation of the vehicle around the $O_{M} Z_{M}$ axis is $T_{z}=F \sin (\psi-\theta) \bar{L}$.

By applying the Euler-Lagrange principle to the model of the mobile manipulator one has:

$$
\begin{aligned}
& \frac{\partial}{\partial t} \frac{\partial L}{\partial \dot{x}}-\frac{\partial L}{\partial x}=F_{X} \\
& \frac{\partial}{\partial t} \frac{\partial L}{\partial \dot{y}}-\frac{\partial L}{\partial y}=F_{Y} \\
& \frac{\partial}{\partial t} \frac{\partial L}{\partial \dot{\psi}}-\frac{\partial L}{\partial \psi}=T_{Z} \\
& \frac{\partial}{\partial t} \frac{\partial L}{\partial \dot{\theta}_{1}}-\frac{\partial L}{\partial \theta_{1}}=T_{1} \\
& \frac{\partial}{\partial t} \frac{\partial L}{\partial \dot{\theta}_{2}}-\frac{\partial L}{\partial \theta_{2}}=T_{2}
\end{aligned}
$$

The aggregate Lagrangian for the model of the mobile manipulator is

$$
\begin{gathered}
L=\frac{1}{2} m_{1}\left(l_{c_{1}} \dot{\theta}_{1}\right)^{2}+\frac{1}{2} I_{1} \dot{\theta}_{1}^{2}+\frac{1}{2} m_{1}\left(V_{x}^{2}+V_{y}^{2}\right)+ \\
+\frac{1}{2} m_{1}\left(l_{c_{1}} \dot{\psi}\right)^{2}+\frac{1}{2} I_{1} \dot{\psi}^{2}+ \\
\frac{1}{2} m_{2}\left[l_{1}^{2} \dot{\theta}_{1}^{2}+l_{c_{1}}^{2}\left(\dot{\theta}_{1}+\dot{\theta}_{2}\right)^{2}+\right. \\
\left.+2 l_{1} l_{c_{2}} \cos \left(\theta_{2}\right) \dot{\theta}_{1}\left(\dot{\theta}_{1}+\dot{\theta}_{2}\right)\right]+ \\
+\frac{1}{2} I_{2}\left(\dot{\theta}_{1}^{2}+\dot{\theta}_{2}^{2}\right)+\frac{1}{2} m_{2}\left(V_{x}^{2}+V_{y}^{2}\right)+ \\
+\frac{1}{2} m_{2}\left\{\left[l_{1}+l_{c_{2}} \cos \left(\theta_{2}\right)\right] \dot{\psi}\right\}^{2}+\frac{1}{2} I_{2} \dot{\psi}^{2} \\
-\left[m_{1} g l_{c_{1}} \cos \left(\theta_{1}\right)\right]-m_{2} g\left[\left(l_{1} \cos \left(\theta_{1}\right)\right)+\right. \\
\left.+l_{c 2} \cos \left(\theta_{1}+\theta_{2}\right)\right]+\frac{1}{2} M\left(\dot{X}^{2}+\dot{Y}^{2}\right)+\frac{1}{2} I_{Z} \dot{\psi}^{2}
\end{gathered}
$$

From the Lagrangian equation given in Eq. (16) one has:

$$
\begin{gathered}
\ddot{X}=\frac{1}{m_{1}+m_{2}+M} F_{X} \\
\ddot{Y}=\frac{1}{m_{1}+m_{2}+M} F_{Y} \\
\ddot{\psi}=\frac{1}{I_{z}+I_{1}+I_{2}+m_{1} l_{c_{1}}^{2}+m_{2}\left(l_{1}+l_{c_{2}} \cos \left(\theta_{2}\right)\right)^{2}} T_{Z}
\end{gathered}
$$

Moreover, about the dynamics of the robotic manipulator one obtains

$$
D_{11} \ddot{\theta}_{1}^{2}+D_{12} \ddot{\theta}_{2}^{2}+h_{1}(\theta, \dot{\theta})+g_{1}(\theta)=T_{1}
$$

where $D_{11}(\theta)=m_{1} l_{c_{1}}^{2}+I_{1}+m_{1} l_{1}^{2}+m_{2} l_{c_{2}}^{2}+$ $2 m_{2} l_{1} l_{c_{2}} \cos \left(\theta_{2}\right)+I_{2}, D_{12}(\theta)=m_{2} l_{c_{2}}^{2}+$ $m_{1} l_{1} l_{c_{2}} \cos \left(\theta_{2}\right), h_{1}(\theta, \dot{\theta})=-m_{2} l_{1} l_{c_{2}} \sin \left(\theta_{2}\right)\left(2 \dot{\theta}_{1}+\dot{\theta}_{2}\right)$ and $g_{1}(\theta)=m_{1} g l_{c_{1}} \sin \left(\theta_{1}\right)+m_{2} g\left[l_{1} \sin \left(\theta_{1}\right)+\right.$ $l_{c_{2}} \sin \left(\theta_{1}+\theta_{2}\right)$.

and also

$$
D_{21} \ddot{\theta}_{1}^{2}+D_{22} \ddot{\theta}_{2}^{2}+h_{2}\left(\theta, \dot{\theta}+g_{2}(\theta)\right)=T_{2}
$$

where $D_{21}(\theta)=m_{2} l_{c_{1}}^{2}+m_{2} l_{1} l_{c_{2}} \cos \left(\theta_{2}\right)$, $D_{22}(\theta)=m_{2} l_{c_{2}}^{2}+I_{2}, h_{2}(\theta \cdot \dot{\theta})=-m_{2} l_{1} l_{c_{2}} \sin \left(\theta_{2}\right) \dot{\theta}_{1}+$ $m_{2}\left(l_{1}+l_{c_{2}} \cos \left(\theta_{2}\right)\right)\left(l_{2} \sin \left(\theta_{2}\right)\right) \dot{\psi}^{2}$, and $g_{2}(\theta)=$ $m_{2} g l_{c_{2}} \sin \left(\theta_{1}+\theta_{2}\right)$.

Thus, about the manipulator attached to the mobile platform one arrives at the following state-space description:

$$
\left(\begin{array}{ll}
D_{11} & D_{12} \\
D_{21} & D_{22}
\end{array}\right)\left(\begin{array}{l}
\ddot{\theta}_{1} \\
\ddot{\theta}_{2}
\end{array}\right)+\left(\begin{array}{l}
h_{1}(\theta, \dot{\theta}) \\
h_{2}(\theta, \dot{\theta})
\end{array}\right)+\left(\begin{array}{l}
g_{1}(\theta) \\
g_{2}(\theta)
\end{array}\right)=\left(\begin{array}{l}
T_{1} \\
T_{2}
\end{array}\right)
$$

By inverting matrix $D(\theta)$, the state-space description of the robotic manipulator can be written as

$$
\begin{aligned}
\left(\begin{array}{c}
\ddot{\theta}_{1} \\
\ddot{\theta}_{2}
\end{array}\right) & =-\frac{1}{D_{11} D_{22}-D_{12} D_{21}}\left(\begin{array}{cc}
D_{22} & -D_{12} \\
-D_{21} & D_{11}
\end{array}\right)\left(\begin{array}{l}
h_{1}(\theta, \dot{\theta}) \\
h_{2}(\theta, \dot{\theta})
\end{array}\right) \\
- & \frac{1}{D_{11} D_{22}-D_{12} D_{21}}\left(\begin{array}{cc}
D_{22} & -D_{12} \\
-D_{21} & D_{11}
\end{array}\right)\left(\begin{array}{l}
g_{1}(\theta) \\
g_{2}(\theta)
\end{array}\right)+ \\
& +\frac{1}{D_{11} D_{22}-D_{12} D_{21}}\left(\begin{array}{cc}
D_{22} & -D_{12} \\
-D_{21} & D_{11}
\end{array}\right)\left(\begin{array}{l}
T_{1} \\
T_{2}
\end{array}\right)
\end{aligned}
$$


The dynamics of the robotic manipulator can be also brought to the following form:

$$
\begin{gathered}
\left(\begin{array}{c}
\ddot{\theta}_{1} \\
\ddot{\theta}_{2}
\end{array}\right)=\left(\begin{array}{l}
-\frac{D_{22} h_{1}-D_{21} h_{2}}{\left(D_{11} D_{22}-D_{12} D_{21}\right)}-\frac{D_{22} g_{1}-D_{21} g_{2}}{\left(D_{11} D_{22}-D_{12} D_{21}\right)} \\
-\frac{-D_{12} h_{1}+D_{11} h_{2}}{\left(D_{11} D_{22}-D_{12} D_{21}\right)}-\frac{-D_{12} g_{1}+D_{11} g_{2}}{\left(D_{11} D_{22}-D_{12} D_{21}\right)}
\end{array}\right)+ \\
+\left(\begin{array}{l}
\frac{D_{22}}{\left(D_{11} D_{22}-D_{12} D_{21}\right)} \frac{-D_{21}}{\left(D_{11} D_{22}-D_{12} D_{21}\right)} \\
\frac{-D_{12}}{\left(D_{11} D_{22}-D_{12} D_{21}\right)}
\end{array}\right)\left(\begin{array}{l}
T_{1} \\
\left(D_{11} D_{22}-D_{12} D_{21}\right)
\end{array}\right)
\end{gathered}
$$

Consequently, the state-space model of the mobile manipulator becomes:

$$
\left(\begin{array}{c}
\ddot{X} \\
\ddot{Y} \\
\ddot{\psi} \\
\ddot{\theta}_{1} \\
\ddot{\theta}_{2}
\end{array}\right)=\left(\begin{array}{c}
\tilde{F}_{1} \\
\tilde{F}_{2} \\
\tilde{F}_{3} \\
\tilde{F}_{4} \\
\tilde{F}_{5}
\end{array}\right)+\left(\begin{array}{ccccc}
g_{11} & 0 & 0 & 0 & 0 \\
0 & g_{22} & 0 & 0 & 0 \\
0 & 0 & g_{33} & 0 & 0 \\
0 & 0 & 0 & g_{44} & g_{45} \\
0 & 0 & 0 & g_{54} & g_{55}
\end{array}\right)\left(\begin{array}{c}
F_{X} \\
F_{Y} \\
T_{Z} \\
T_{1} \\
T_{2}
\end{array}\right)
$$

where $\tilde{F}_{1}=0, \quad \tilde{F}_{2}=0, \tilde{F}_{3}=0$, $\tilde{F}_{4}=-\frac{D_{22} h_{1}-D_{21} h_{2}}{\left(D_{11} D_{22}-D_{12} D_{21}\right)}-\frac{D_{22} g_{1}-D_{21} g_{2}}{\left(D_{11} D_{22}-D_{12} D_{21}\right)}$, $\tilde{F}_{5}=-\frac{-D_{12} h_{1}+D_{11} h_{2}}{\left(D_{11} D_{22}-D_{12} D_{21}\right)}-\frac{-D_{12} g_{1}+D_{11} g_{2}}{\left(D_{11} D_{22}-D_{12} D_{21}\right)}$,

while it also holds that $g_{11}=\frac{1}{m_{1}+m_{2}+M}, g_{22}=$ $\frac{1}{m_{1}+m_{2}+M}, g_{33}=\frac{1}{I_{Z}+I_{1}+I_{2}+m_{1} l_{c_{1}}^{2}+m_{2}\left(l_{1}+l_{c_{2}} \cos \left(\theta_{2}\right)\right)^{2}}$, $g_{44}=\frac{D_{22}}{\left(D_{11} D_{22}-D_{12} D_{21}\right)}, g_{45}=\frac{-D_{21}}{\left(D_{11} D_{22}-D_{12} D_{21}\right)}$, $g_{54}=\frac{-D_{12}}{\left(D_{11} D_{22}-D_{12} D_{21}\right)}$, and $g_{55}=\frac{D_{11}}{\left(D_{11} D_{22}-D_{12} D_{21}\right)}$.

\section{Differential Flatness Properties of the Model of the Mobile Manipulator}

It will be proven that the dynamic model of the mobile manipulator is a differentially flat one, which implies that all its state variables and its control inputs can be expressed as differential functions of a specific subset of its state vector elements which are known as flat outputs of the system. The following state variables of the mobile manipulator are defined: $x_{1}=X, x_{2}=\dot{X}$, $x_{3}=Y, x_{4}=\dot{Y}, x_{5}=\psi, x_{6}=\dot{\psi}, x_{7}=\theta_{1}, x_{8}=\dot{\theta}_{1}$, $x_{9}=\theta_{2}, x_{10}=\dot{\theta}_{2}$. Additionally, the control inputs of the robotic system are taken to be $u_{1}=F_{X}, u_{2}=F_{Y}$, $u_{3}=T_{Z}, u_{4}=T_{1}$ and $u_{5}=T_{2}$. Thus, the following state-space description is obtained:

$$
\left(\begin{array}{c}
\dot{x}_{1} \\
\dot{x}_{2} \\
\dot{x}_{3} \\
\dot{x}_{4} \\
\dot{x}_{5} \\
\dot{x}_{6} \\
\dot{x}_{7} \\
\dot{x}_{8} \\
\dot{x}_{9} \\
\dot{x}_{10}
\end{array}\right)=\left(\begin{array}{c}
x_{2} \\
\tilde{F}_{1} \\
x_{4} \\
\tilde{F}_{2} \\
x_{6} \\
\tilde{F}_{3} \\
x_{8} \\
\tilde{F}_{4} \\
x_{10} \\
\tilde{F}_{5}
\end{array}\right)+\left(\begin{array}{ccccc}
0 & 0 & 0 & 0 & 0 \\
g_{11} & 0 & 0 & 0 & 0 \\
0 & 0 & 0 & 0 & 0 \\
0 & g_{22} & 0 & 0 & 0 \\
0 & 0 & 0 & 0 & 0 \\
0 & 0 & g_{33} & 0 & 0 \\
0 & 0 & 0 & 0 & 0 \\
0 & 0 & 0 & g_{44} & g_{45} \\
0 & 0 & 0 & 0 & 0 \\
0 & 0 & 0 & g_{54} & g_{55}
\end{array}\right)\left(\begin{array}{l}
u_{1} \\
u_{2} \\
u_{3} \\
u_{4} \\
u_{5}
\end{array}\right)
$$

The vector of the system's flat outputs is $x_{f}=$ $\left[x_{1}, x_{3}, x_{5}, x_{7}, x_{9}\right]^{T}$. It holds that $x_{2}=\dot{x}_{1}, x_{4}=\dot{x}_{3}$, $x_{6}=\dot{x}_{5}, x_{8}=\dot{x}_{7}$ and $x_{10}=\dot{x}_{9}$. Therefore, all state vector elements of the mobile manipulator can be written as differential functions of the flat output vector. Moreover, from the second row of Eq. (31) one has

$$
u_{1}=\frac{1}{g_{11}} \dot{x}_{2}-\tilde{F}_{1} \Rightarrow u_{1}=q_{1}\left(x_{f}, \dot{x}_{f}\right)
$$

From the fourth row of Eq. (31) one has

$$
u_{2}=\frac{1}{g_{22}} \dot{x}_{4}-\tilde{F}_{2} \Rightarrow u_{2}=q_{2}\left(x_{f}, \dot{x}_{f}\right)
$$

From the sixth row of Eq. (31) one has

$$
u_{3}=\frac{1}{g_{33}} \dot{x}_{6}-\tilde{F}_{3} \Rightarrow u_{3}=q_{3}\left(x_{f}, \dot{x}_{f}\right)
$$

From the eight and tenth rows of Eq. (31) one has

$$
\begin{gathered}
\left(\begin{array}{c}
u_{5} \\
u_{6}
\end{array}\right)=D\left(\begin{array}{c}
\dot{x}_{8} \\
\dot{x}_{10}
\end{array}\right)+\left(\begin{array}{l}
h_{1} \\
h_{2}
\end{array}\right)+\left(\begin{array}{l}
g_{1} \\
g_{2}
\end{array}\right) \\
\Rightarrow \begin{array}{l}
u_{4}=q_{4}\left(x_{f}, \dot{x}_{f}\right) \\
u_{5}=q_{5}\left(x_{f}, \dot{x}_{f}\right)
\end{array}
\end{gathered}
$$

Consequently the control inputs of the mobile manipulator are also written as differential functions of the flat outputs vector and the system is a differentially flat one.

\section{Design of a Flatness-based Controller for the Mo- bile Manipulator}

The dynamic model of the mobile manipulator has been given in Eq. (31) The following control inputs are applied:

$$
\begin{aligned}
& u_{1}=\frac{1}{g_{11}}\left[\ddot{x}_{1}^{d}-\tilde{F}_{1}-k_{1}^{1}\left(\dot{x}_{1}-\dot{x}_{1}^{d}\right)-k_{2}^{1}\left(x_{1}-x_{1}^{d}\right)\right] \\
& u_{2}=\frac{1}{g_{22}}\left[\ddot{x}_{3}^{d}-\tilde{F}_{2}-k_{1}^{2}\left(\dot{x}_{3}-\dot{x}_{3}^{d}\right)-k_{2}^{2}\left(x_{3}-x_{3}^{d}\right)\right]
\end{aligned}
$$

$$
u_{3}=\frac{1}{g_{33}}\left[\ddot{x}_{5}^{d}-\tilde{F}_{3}-k_{1}^{3}\left(\dot{x}_{5}-\dot{x}_{5}^{d}\right)-k_{2}^{3}\left(x_{5}-x_{5}^{d}\right)\right]
$$

$$
\begin{gathered}
\left(\begin{array}{l}
u_{4} \\
u_{5}
\end{array}\right)=\left(\begin{array}{ll}
g_{44} & g_{45} \\
g_{54} & g_{55}
\end{array}\right)^{-1}\left\{\left(\begin{array}{l}
\ddot{x}_{7}^{d} \\
\ddot{x}_{9}^{d}
\end{array}\right)-\right. \\
\left.-\left(\begin{array}{c}
\tilde{F}_{4} \\
\tilde{F}_{5}
\end{array}\right)-\left(\begin{array}{l}
k_{1}^{4}\left(\dot{x}_{7}-\dot{x}_{7}^{d}\right)+k_{2}^{4}\left(x_{7}-x_{7}^{d}\right) \\
k_{1}^{5}\left(\dot{x}_{9}-\dot{x}_{9}^{d}\right)+k_{2}^{5}\left(x_{9}-x_{9}^{d}\right)
\end{array}\right)\right\}
\end{gathered}
$$


By applying the previous control inputs to the statespace model of Eq. (31) one gets:

$$
\begin{aligned}
& \left(\ddot{x}_{1}-\ddot{x}_{1}^{d}\right)+k_{1}^{1}\left(\dot{x}_{1}-\dot{x}_{1}^{d}\right)+k_{2}^{1}\left(x_{1}-x_{1}^{d}\right)=0 \\
& \left(\ddot{x}_{3}-\ddot{x}_{3}^{d}\right)+k_{1}^{2}\left(\dot{x}_{3}-\dot{x}_{3}^{d}\right)+k_{2}^{2}\left(x_{3}-x_{3}^{d}\right)=0 \\
& \left(\ddot{x}_{5}-\ddot{x}_{5}^{d}\right)+k_{1}^{3}\left(\dot{x}_{5}-\dot{x}_{5}^{d}\right)+k_{2}^{3}\left(x_{5}-x_{5}^{d}\right)=0 \\
& \left(\ddot{x}_{7}-\ddot{x}_{7}^{d}\right)+k_{1}^{4}\left(\dot{x}_{7}-\dot{x}_{7}^{d}\right)+k_{2}^{4}\left(x_{7}-x_{7}^{d}\right)=0 \\
& \left(\ddot{x}_{9}-\ddot{x}_{9}^{d}\right)+k_{1}^{5}\left(\dot{x}_{9}-\dot{x}_{9}^{d}\right)+k_{2}^{5}\left(x_{9}-x_{9}^{d}\right)=0
\end{aligned}
$$

By defining the state variables' tracking error as $e_{i}=$ $x_{i}-x_{i}^{d}, i=1,2, \cdots, 5$ one obtains the tracking error dynamics through the following equations:

$$
\begin{aligned}
& \ddot{e}_{1}+k_{1}^{1} \dot{e}_{1}+k_{2}^{1} e_{1}=0 \\
& \ddot{e}_{2}+k_{1}^{2} \dot{e}_{2}+k_{2}^{2} e_{2}=0 \\
& \ddot{e}_{3}+k_{1}^{3} \dot{e}_{3}+k_{2}^{3} e_{3}=0 \\
& \ddot{e}_{4}+k_{1}^{4} \dot{e}_{4}+k_{2}^{4} e_{4}=0 \\
& \ddot{e}_{5}+k_{1}^{5} \dot{e}_{5}+k_{2}^{5} e_{1}=0
\end{aligned}
$$

Next, by selecting the feedback gains $\left(k_{1}^{i}, k_{2}^{i}\right), i=$ $1,2, \cdots, 5$ such that the characteristic polynomials which are associated with the aforementioned differential equations to be Hurwitz stable, one has that

$$
\begin{gathered}
\lim _{t \rightarrow \infty} e_{i}=0, \quad i=1,2, \cdots, 5 \Rightarrow \\
\lim _{t \rightarrow \infty} x_{i}=x_{i}^{d}, \quad i=1,2, \cdots, 5
\end{gathered}
$$

\section{Design of a Flatness-based Disturbances Estima- tor}

Next, the dynamic model of the mobile manipulator is considered to be affected by additive input disturbances:

$$
\left(\begin{array}{c}
\dot{x}_{1} \\
\dot{x}_{2} \\
\dot{x}_{3} \\
\dot{x}_{4} \\
\dot{x}_{5} \\
\dot{x}_{6} \\
\dot{x}_{7} \\
\dot{x}_{8} \\
\dot{x}_{9} \\
\dot{x}_{10}
\end{array}\right)=\left(\begin{array}{c}
x_{2} \\
\tilde{F}_{1} \\
x_{4} \\
\tilde{F}_{2} \\
x_{6} \\
\tilde{F}_{3} \\
x_{8} \\
\tilde{F}_{4} \\
x_{10} \\
\tilde{F}_{5}
\end{array}\right)+\left(\begin{array}{ccccc}
0 & 0 & 0 & 0 & 0 \\
g_{11} & 0 & 0 & 0 & 0 \\
0 & 0 & 0 & 0 & 0 \\
0 & g_{22} & 0 & 0 & 0 \\
0 & 0 & 0 & 0 & 0 \\
0 & 0 & g_{33} & 0 & 0 \\
0 & 0 & 0 & 0 & 0 \\
0 & 0 & 0 & g_{44} & g_{45} \\
0 & 0 & 0 & 0 & 0 \\
0 & 0 & 0 & g_{54} & g_{55}
\end{array}\right)\left(\begin{array}{l}
u_{1} \\
u_{2} \\
u_{3} \\
u_{4} \\
u_{5}
\end{array}\right)+\left(\begin{array}{c}
0 \\
d_{1} \\
0 \\
d_{2} \\
0 \\
d_{3} \\
0 \\
d_{4} \\
0 \\
d_{5}
\end{array}\right)
$$

Using the previous state-space model the following virtual control inputs are defined: $v_{1}=\tilde{F}_{1}+g_{11} u_{1}, v_{2}=\tilde{F}_{2}+g_{22} u_{2}, v_{3}=\tilde{F}_{3}+g_{33} u_{3}$, $v_{4}=\tilde{F}_{4}+g_{44} u_{4}+g_{45} u_{5}$, and $v_{5}=\tilde{F}_{5}+g_{54} u_{4}+g_{55} u_{5}$. Equivalently, the dynamics of the mobile manipulator under additive input disturbances is written as: $\ddot{x}_{1}=v_{1}+d_{1}, \ddot{x}_{2}=v_{2}+d_{2}, \ddot{x}_{3}=v_{3}+d_{3}$, $\ddot{x}_{4}=v_{4}+d_{4}, \ddot{x}_{5}=v_{5}+d_{5}$.
The disturbances terms $d_{i}, i=1,2, \cdots, 5$ are considered to be described by the associated 2 nd order time-derivative and by the related initial conditions. This is reasonable, because every function $f$ can be equivalently represented by its $n$-th order timederivative $f^{(n)}(t)$ and by the related initial conditions $f^{(i)}(0), \quad i=1,2, \cdots, n-1$. However, since estimation of such functions is going to be performed with the use of Kalman Filtering, prior knowledge about initial conditions becomes obsolete.

The second-order derivative of the aforementioned disturbance inputs is given by $\ddot{d}_{i}=f_{d_{i}}, i=1,2, \cdots, 5$. Next, the state variables and their time-derivatives are denoted as additional state variables of the model of the mobile manipulator. This results into the following state-space description of the system: $z_{1}=x_{1}, z_{2}=\dot{x}_{1}$, $z_{3}=x_{2}, z_{4}=\dot{x}_{2}, z_{5}=x_{3}, z_{6}=\dot{x}_{3}, z_{7}=x_{4}, z_{8}=\dot{x}_{4}$, $z_{9}=x_{5}, z_{10}=\dot{x}_{5}, z_{11}=d_{1}, z_{12}=\dot{d}_{1}, z_{13}=d_{2}$, $z_{14}=\dot{d}_{2}, z_{15}=d_{3}, z_{16}=\dot{d}_{3}, z_{17}=d_{4}, z_{18}=\dot{d}_{4}$ and $z_{19}=d_{5}, z_{20}=\dot{d}_{5}$.

The model of the mobile manipulator can be written in the following concise state-space form:

$$
\begin{gathered}
\dot{z}=A z+B \tilde{v} \\
z_{m}=C z
\end{gathered}
$$

where vector $\quad \tilde{v} \quad=$ $\left[u_{1}, u_{2}, u_{3}, u_{4}, u_{5}, f_{d_{1}}, f_{d_{2}}, f_{d_{3}}, f_{d_{4}}, f_{d_{5}}\right]^{T} \quad$ while matrices $A \in R^{20 \times 20}, B \in R^{20 \times 10}$ and $C \in R^{5 \times 20}$ are given by

$$
A=\left(\begin{array}{llllllllllllllllllll}
0 & 1 & 0 & 0 & 0 & 0 & 0 & 0 & 0 & 0 & 0 & 0 & 0 & 0 & 0 & 0 & 0 & 0 & 0 & 0 \\
0 & 0 & 0 & 0 & 0 & 0 & 0 & 0 & 0 & 0 & 1 & 0 & 0 & 0 & 0 & 0 & 0 & 0 & 0 & 0 \\
0 & 0 & 0 & 1 & 0 & 0 & 0 & 0 & 0 & 0 & 0 & 0 & 0 & 0 & 0 & 0 & 0 & 0 & 0 & 0 \\
0 & 0 & 0 & 0 & 0 & 0 & 0 & 0 & 0 & 0 & 0 & 0 & 1 & 0 & 0 & 0 & 0 & 0 & 0 & 0 \\
0 & 0 & 0 & 0 & 0 & 1 & 0 & 0 & 0 & 0 & 0 & 0 & 0 & 0 & 0 & 0 & 0 & 0 & 0 & 0 \\
0 & 0 & 0 & 0 & 0 & 0 & 0 & 0 & 0 & 0 & 0 & 0 & 0 & 0 & 1 & 0 & 0 & 0 & 0 & 0 \\
0 & 0 & 0 & 0 & 0 & 0 & 0 & 1 & 0 & 0 & 0 & 0 & 0 & 0 & 0 & 0 & 0 & 0 & 0 & 0 \\
0 & 0 & 0 & 0 & 0 & 0 & 0 & 0 & 0 & 0 & 0 & 0 & 0 & 0 & 0 & 0 & 1 & 0 & 0 & 0 \\
0 & 0 & 0 & 0 & 0 & 0 & 0 & 0 & 0 & 1 & 0 & 0 & 0 & 0 & 0 & 0 & 0 & 0 & 0 & 0 \\
0 & 0 & 0 & 0 & 0 & 0 & 0 & 0 & 0 & 0 & 0 & 0 & 0 & 0 & 0 & 0 & 0 & 0 & 1 & 0 \\
0 & 0 & 0 & 0 & 0 & 0 & 0 & 0 & 0 & 0 & 0 & 1 & 0 & 0 & 0 & 0 & 0 & 0 & 0 & 0 \\
0 & 0 & 0 & 0 & 0 & 0 & 0 & 0 & 0 & 0 & 0 & 0 & 0 & 0 & 0 & 0 & 0 & 0 & 0 & 0 \\
0 & 0 & 0 & 0 & 0 & 0 & 0 & 0 & 0 & 0 & 0 & 0 & 0 & 1 & 0 & 0 & 0 & 0 & 0 & 0 \\
0 & 0 & 0 & 0 & 0 & 0 & 0 & 0 & 0 & 0 & 0 & 0 & 0 & 0 & 0 & 0 & 0 & 0 & 0 & 0 \\
0 & 0 & 0 & 0 & 0 & 0 & 0 & 0 & 0 & 0 & 0 & 0 & 0 & 0 & 0 & 1 & 0 & 0 & 0 & 0 \\
0 & 0 & 0 & 0 & 0 & 0 & 0 & 0 & 0 & 0 & 0 & 0 & 0 & 0 & 0 & 0 & 0 & 0 & 0 & 0 \\
0 & 0 & 0 & 0 & 0 & 0 & 0 & 0 & 0 & 0 & 0 & 0 & 0 & 0 & 0 & 0 & 0 & 1 & 0 & 0 \\
0 & 0 & 0 & 0 & 0 & 0 & 0 & 0 & 0 & 0 & 0 & 0 & 0 & 0 & 0 & 0 & 0 & 0 & 0 & 0 \\
0 & 0 & 0 & 0 & 0 & 0 & 0 & 0 & 0 & 0 & 0 & 0 & 0 & 0 & 0 & 0 & 0 & 0 & 0 & 1 \\
0 & 0 & 0 & 0 & 0 & 0 & 0 & 0 & 0 & 0 & 0 & 0 & 0 & 0 & 0 & 0 & 0 & 0 & 0 & 0
\end{array}\right)
$$




$$
\begin{aligned}
& B=\left(\begin{array}{llllllllll}
0 & 0 & 0 & 0 & 0 & 0 & 0 & 0 & 0 & 0 \\
1 & 0 & 0 & 0 & 0 & 0 & 0 & 0 & 0 & 0 \\
0 & 0 & 0 & 0 & 0 & 0 & 0 & 0 & 0 & 0 \\
0 & 1 & 0 & 0 & 0 & 0 & 0 & 0 & 0 & 0 \\
0 & 0 & 0 & 0 & 0 & 0 & 0 & 0 & 0 & 0 \\
0 & 0 & 1 & 0 & 0 & 0 & 0 & 0 & 0 & 0 \\
0 & 0 & 0 & 0 & 0 & 0 & 0 & 0 & 0 & 0 \\
0 & 0 & 0 & 1 & 0 & 0 & 0 & 0 & 0 & 0 \\
0 & 0 & 0 & 0 & 0 & 0 & 0 & 0 & 0 & 0 \\
0 & 0 & 0 & 0 & 1 & 0 & 0 & 0 & 0 & 0 \\
0 & 0 & 0 & 0 & 0 & 0 & 0 & 0 & 0 & 0 \\
0 & 0 & 0 & 0 & 0 & 1 & 0 & 0 & 0 & 0 \\
0 & 0 & 0 & 0 & 0 & 0 & 0 & 0 & 0 & 0 \\
0 & 0 & 0 & 0 & 0 & 0 & 1 & 0 & 0 & 0 \\
0 & 0 & 0 & 0 & 0 & 0 & 0 & 0 & 0 & 0 \\
0 & 0 & 0 & 0 & 0 & 0 & 0 & 1 & 0 & 0 \\
0 & 0 & 0 & 0 & 0 & 0 & 0 & 0 & 0 & 0 \\
0 & 0 & 0 & 0 & 0 & 0 & 0 & 0 & 0 & 1 \\
0 & 0 & 0 & 0 & 0 & 0 & 0 & 0 & 0 & 0
\end{array}\right)
\end{aligned}
$$

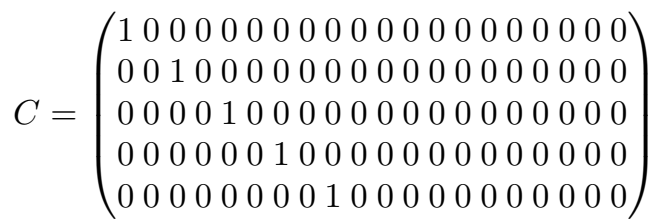

The associated state-vector and disturbances estimation problem can be solved by considering a disturbance observer in the form:

$$
\begin{gathered}
\hat{z}=A_{o} \hat{z}+B_{o} v+K_{f}\left[z_{m}-\hat{z}_{m}\right] \\
\hat{z}_{m}=C_{o} \hat{z}
\end{gathered}
$$

where $A_{o}=A, B_{o}=B$ and $B_{o}$ is obtained from $B$ after zeroing all elements in its last 5 columns. The estimator's gain $K_{f}$ is computed by applying the Kalman Filter's recursion. To this end, matrices $A_{o}$, $B_{o}$ and $C_{o}$ are substituted by their discrete-time equivalents $A_{d}, B_{d}, C_{d}$ after using common discretization methods. The Kalman Filter's recursion consists of a measurement-update stage and of a time-update stage:

measurement update:

$$
\begin{gathered}
K_{f}(k)=P^{-}(k) C_{d}^{T}\left[C_{d} P^{-}(k) C_{d}^{T}+R\right]^{-1} \\
\hat{z}(k)=\hat{z}^{-}(k)+K_{f}(k)\left[z_{m}-\hat{z}_{m}\right] \\
P(k)=P^{-}(k)-K_{f}(k) C_{d} P^{-}(k)
\end{gathered}
$$

time update:

$$
\begin{gathered}
P^{-}(k+1)=A_{d} P(k) A_{d}^{T}+Q \\
\hat{z}^{-}(k+1)=A_{d} \hat{z}(k)+B_{d} v(k)
\end{gathered}
$$

By identifying the unknown disturbance terms $d_{i}, i-$ $1,2, \cdots, 5$ the control system of the robotic unit is modified as follows:

$$
\begin{gathered}
u_{1}=\frac{1}{g_{11}}\left[\ddot{x}_{1}^{d}-\tilde{F}_{1}-k_{1}^{1}\left(\dot{x}_{1}-\dot{x}_{1}^{d}\right)-k_{2}^{1}\left(x_{1}-x_{1}^{d}\right)-\hat{d}_{1}\right] \\
u_{2}=\frac{1}{g_{22}}\left[\ddot{x}_{3}^{d}-\tilde{F}_{2}-k_{1}^{2}\left(\dot{x}_{3}-\dot{x}_{3}^{d}\right)-k_{2}^{2}\left(x_{3}-x_{3}^{d}\right)\right] \\
u_{3}=\frac{1}{g_{33}}\left[\ddot{x}_{5}^{d}-\tilde{F}_{3}-k_{1}^{3}\left(\dot{x}_{5}-\dot{x}_{5}^{d}\right)-k_{2}^{3}\left(x_{5}-x_{5}^{d}\right)-\hat{d}_{3}\right] \\
\left(\begin{array}{l}
u_{4} \\
u_{5}
\end{array}\right)=\left(\begin{array}{l}
g_{44} g_{45} \\
g_{54} g_{55}
\end{array}\right)^{-1}\left\{\left(\begin{array}{l}
\ddot{x}_{7}^{d} \\
\ddot{x}_{9}^{d}
\end{array}\right)-\left(\begin{array}{l}
\tilde{F}_{4} \\
\tilde{F}_{5}
\end{array}\right)-\right. \\
\left.-\left(\begin{array}{l}
k_{1}^{4}\left(\dot{x}_{7}-\dot{x}_{7}^{d}\right)+k_{2}^{4}\left(x_{7}-x_{7}^{d}\right) \\
k_{1}^{5}\left(\dot{x}_{9}-\dot{x}_{9}^{d}\right)+k_{2}^{5}\left(x_{9}-x_{9}^{d}\right)
\end{array}\right)-\left(\begin{array}{l}
\hat{d}_{4} \\
\hat{d}_{5}
\end{array}\right)\right\}
\end{gathered}
$$

\section{Simulation Tests}

The performance of the proposed differential flatness theory-based scheme for control and state estimation in mobile manipulators has been confirmed through simulation experiments. The Derivative-free nonlinear Kalman Filter has been redesigned as a disturbances estimator, thus allowing for simultaneous estimation of both the non-measurable state variables of the robotic system and of the additive perturbation terms that were affecting it. The measured state vector elements where $x_{1}=X$ that is the displacement of the robotic vehicle along the $X$ axis, $x_{2}=Y$ that is the displacement of the robotic vehicle along the $Y$ axis, $\psi$ that is the rotation angle of the vehicle around the $Z$ axis, $\theta_{1}$ the turn angle of the first joint of the robotic manipulator, and $\theta_{2}$ that is the turn angle of the second joint of the robotic manipulator. The obtained results are depicted in Fig. 3 to Fig. 18. The real value of the state variables of the robotic system is printed in blue, the estimated value is plotted in green while the associated reference setpoints are shown in red.

The advantages of using a global linearization-based control method for the dynamic model of the mobile manipulator comprising a four-wheels ground vehicle and a two-DOF robotic manipulator are outlined as follows: (i) the transformation that is performed on the robotic system's state-space model is an exact one and does not introduce any modelling errors (ii) by expressing the dynamic model of the mobile manipulator into the linear canonical form it is assured that the separation principle holds and that the design of the controller can be solved independently from the design of the state-observer, (iii) by using the Kalman Filter as a disturbance observer the estimation and compensation of perturbation terms that affect the mobile manipulator's model is achieved and 
thus the robustness of the control scheme is improved (iv) The robustness properties of the control method are equivalent to those of LQG (Linear Quadratic Gaussian) control, (v) by using the Kalman Filter as a disturbance observer it is assured that the optimality of the estimation performed by the Kalman Filter is retained.

It is noted that by finding control inputs $u_{1}, u_{2}$ and $u_{3}$ the previously analysed procedure computed actually the forces $F_{X}, F_{Y}$ and the torque $T_{Z}$ which are exerted on the mobile robot and which define the motion performed by the mobile manipulator. To find also the real control inputs applied on the mobile robot, that is the propulsion force $F$ of its motor, the turn angle $\theta$ of its steering wheels and the braking force $F_{b}$ applied to the rear wheels of the vehicle, the following relations are used:

$$
\begin{gathered}
F_{X}=F \cos (\psi-\theta)-F_{b} \cos (\psi) \\
F_{Y}=F \sin (\psi-\theta)-F_{b} \sin (\psi) \\
T_{Z}=F \sin (\psi-\theta) \bar{L}
\end{gathered}
$$

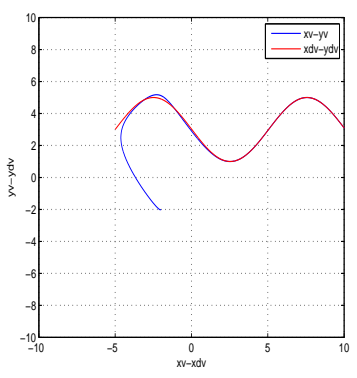

(a)

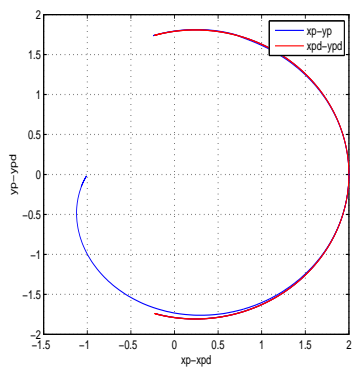

(b)
Figure 3. Test 1: (a) Tracking of reference path (red line) by the robotic vehicle (blue line), (b) Tracking of reference path (red line) by the end-effector of the robotic arm (blue line)

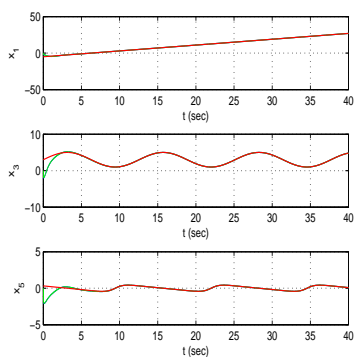

(a)
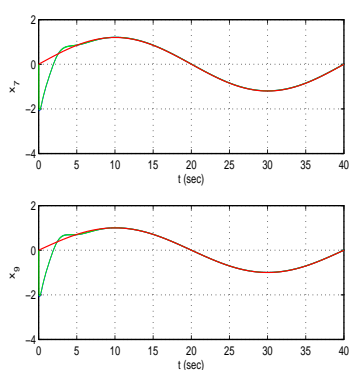

(b)
Figure 4. Test 1: (a) Convergence of state variables $x_{1}=X, x_{3}=$ $Y$ and $x_{5}=\psi$ of the robotic vehicle to setpoints (b) Convergence of states $x_{7}=\theta_{1}$, and $x_{9}=\theta_{2}$ of the robotic arm to setpoints
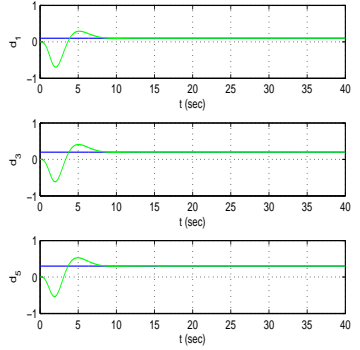

(a)
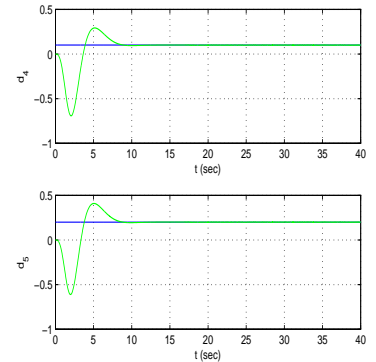

(b)
Figure 5. Test 1: (a) Estimation of the disturbance inputs $d_{1}, d_{2}$ and $d_{3}$ affecting the robotic vehicle with the use of a Kalman Filter-based disturbance observer (b) Estimation of the disturbance inputs $d_{4}$ and $d_{5}$ affecting the robotic arm. with the use of a Kalman Filter-based disturbance observer
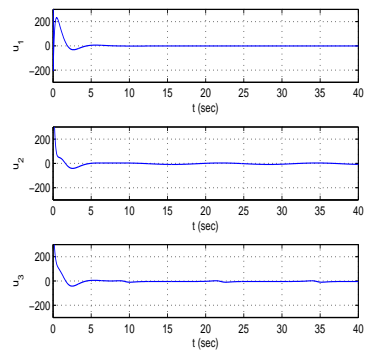

(a)
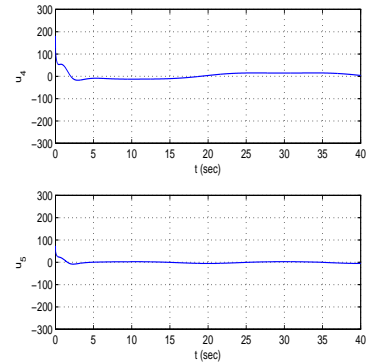

(b)
Figure 6. Test 1: (a) Control inputs $u_{1}, u_{2}$ and $u_{3}$ applied to the robotic vehicle (b) Control inputs $u_{4}$ and $u_{5}$ applied to the robotic arm

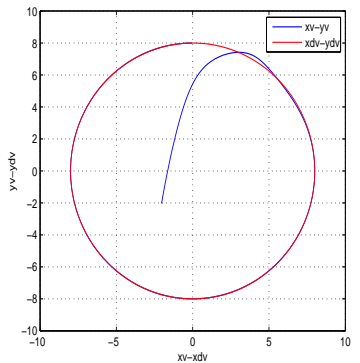

(a)

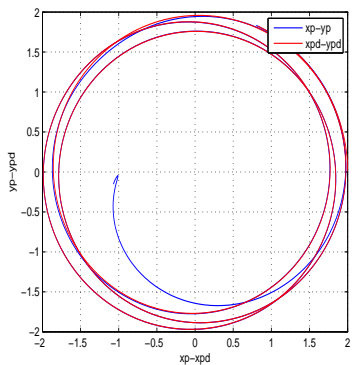

(b)
Figure 7. Test 2: (a) Tracking of reference path (red line) by the robotic vehicle (blue line), (b) Tracking of reference path (red line) by the end-effector of the robotic arm (blue line) 

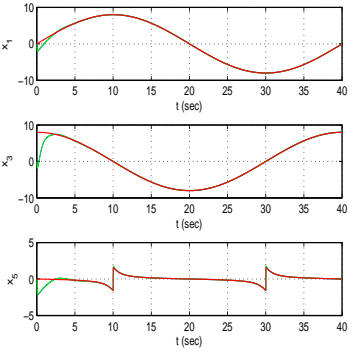

(a)
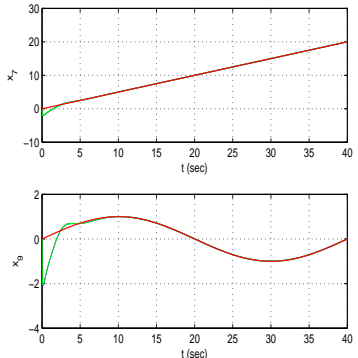

(b)

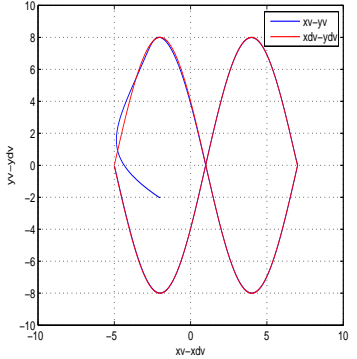

(a)

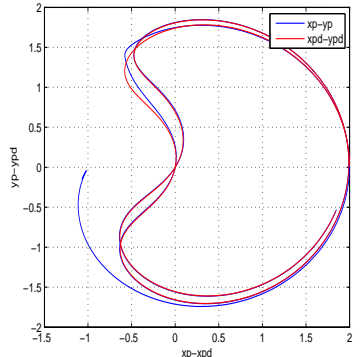

(b)

Figure 8. Test 2: (a) Convergence of state variables $x_{1}=X, x_{3}=$ $Y$ and $x_{5}=\psi$ of the robotic vehicle to setpoints (b) Convergence of state variables $x_{7}=\theta_{1}$, and $x_{9}=\theta_{2}$ of the robotic arm to setpoints
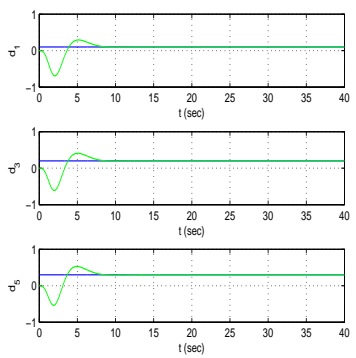

(a)
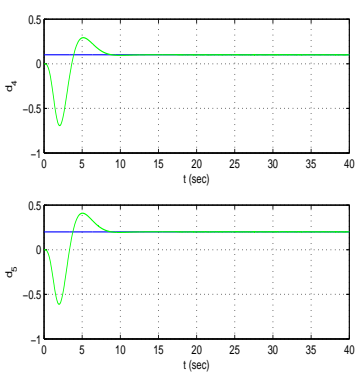

(b)

Figure 9. Test 2: (a) Estimation of the disturbances $d_{1}, d_{2}$ and $d_{3}$ at the robotic vehicle. with the use of a Kalman Filter-based disturbance observer (b) Estimation of the disturbance inputs $d_{4}$ and $d_{5}$ at the robotic arm
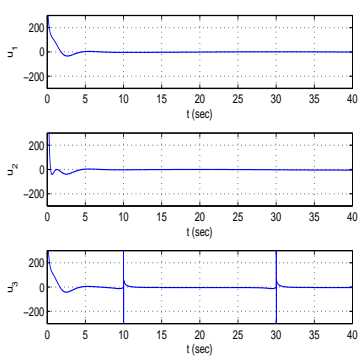

(a)
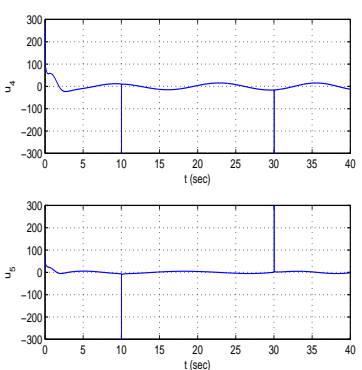

(b)
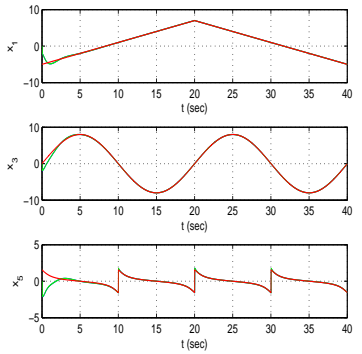

(a)
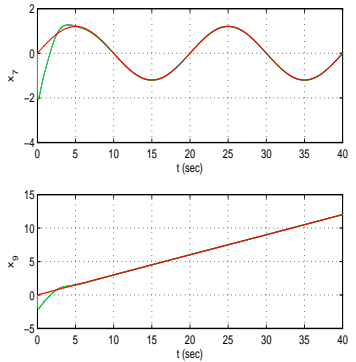

(b)

Figure 12. Test 3: (a) Convergence of state variables $x_{1}=X$, $x_{3}=Y$ and $x_{5}=\psi$ of the robotic vehicle to setpoints (b) Convergence of state variables $x_{7}=\theta_{1}$, and $x_{9}=\theta_{2}$ of the robotic arm to setpoints
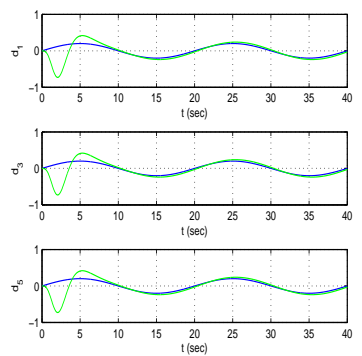

(a)
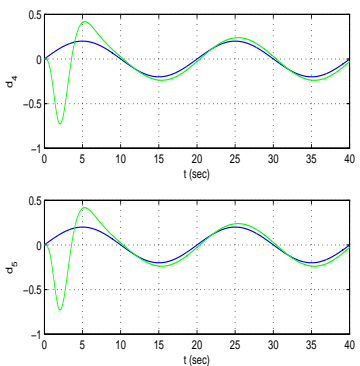

(b)

Figure 10. Test 2: (a) Control inputs $u_{1}, u_{2}, u_{3}$ applied to the robotic vehicle. (b) Control inputs $u_{4}, u_{5}$ applied to the robotic arm

Figure 13. Test 3: (a) Estimation of the disturbance inputs $d_{1}, d_{2}$ and $d_{3}$ affecting the robotic vehicle, with the use of a Kalman Filterbased disturbance observer (b) Estimation of the disturbance inputs $d_{4}$ and $d_{5}$ affecting the robotic arm. with the use of a Kalman Filter-based disturbance observer 

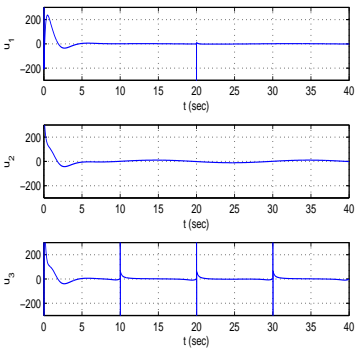

(a)
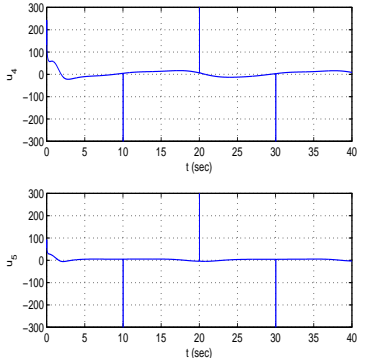

(b)
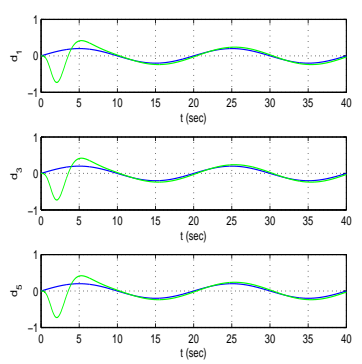

(a)
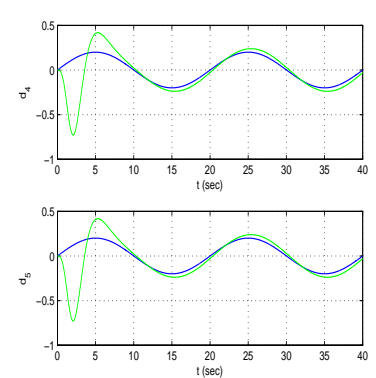

(b)
Figure 14. Test 3: (a) Control inputs $u_{1}, u_{2}$ and $u_{3}$ applied to the robotic vehicle. (b) Control inputs $u_{4}$ and $u_{5}$ applied to the robotic arm

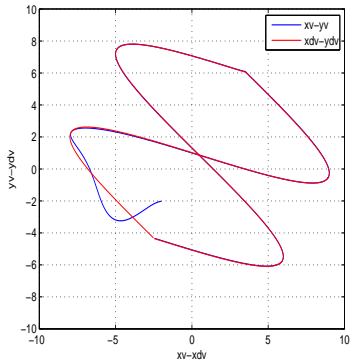

(a)

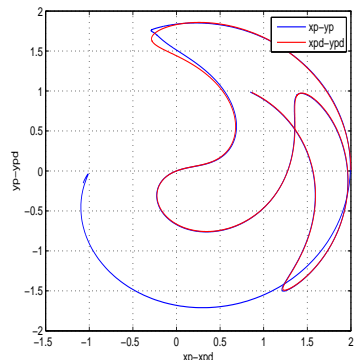

(b)
Figure 15. Test 4: (a) Tracking of reference path (red line) by the robotic vehicle (blue line), (b) Tracking of reference path (red line) by the end-effector of the robotic arm (blue line)

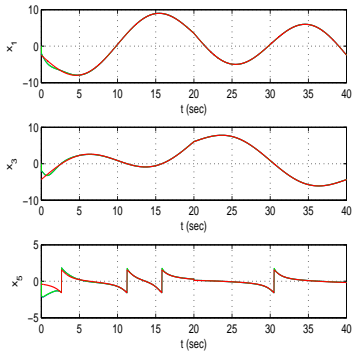

(a)
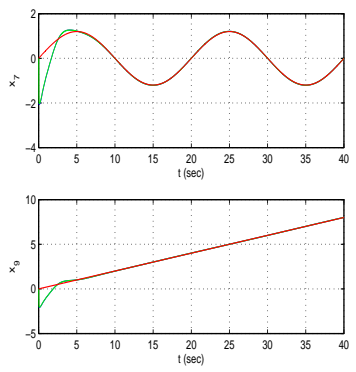

(b)
Figure 16. Test 4: (a) Convergence of state variables $x_{1}=X$, $x_{3}=Y$ and $x_{5}=\psi$ of the robotic vehicle to their reference setpoints (b) Convergence of state variables $x_{7}=\theta_{1}$, and $x_{9}=\theta_{2}$ of the robotic arm to their reference setpoints
Figure 17. Test 4: (a) Estimation of the disturbance inputs $d_{1}, d_{2}$ and $d_{3}$ affecting the robotic vehicle, with the use of a Kalman Filterbased disturbance observer (b) Estimation of the disturbance inputs $d_{4}$ and $d_{5}$ affecting the robotic arm. with the use of a Kalman Filter-based disturbance observer
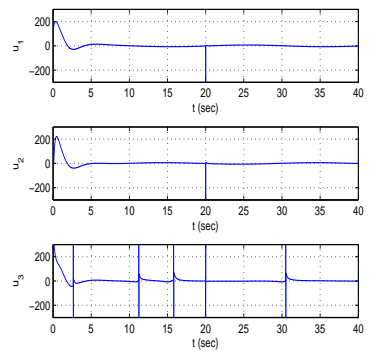

(a)
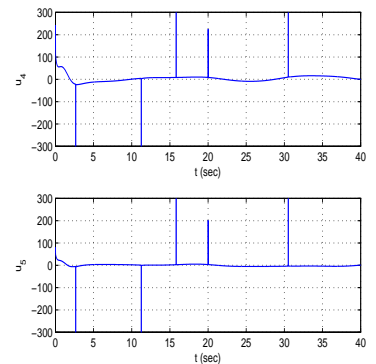

(b)
Figure 18. Test 4: (a) Control inputs $u_{1}, u_{2}$ and $u_{3}$ applied to the robotic vehicle. (b) Control inputs $u_{4}$ and $u_{5}$ applied to the robotic arm

The model of the mobile manipulator which is presented in this manuscript is an indicative example of a multi-body system that satisfies differential flatness properties and on which both differential flatness theorybased control and differential flatness theory-based filtering techniques can be used. Besides, it is a cyberphysical system because filtering techniques are used to estimate missing sensorial information that is needed for implementing state-feedback control. The article's approach for the joint control and state estimation problem of mobile manipulators is outlined as follows: (1) By proving that differential flatness properties hold, then it can be assured that such systems can be transformed into the input-output linearized form which is both controllable and observable, (ii) it is shown that for the inputoutput linearized form, or equivalently for the canonical 
Brunovsky form of such systems the solution of the related control and state estimation problems can be acomplished with the use of linear control and filtering techniques.

\section{Conclusions}

A differential flatness theory-based method has been proposed for control and state estimation of mobile manipulators. It has been proven that the dynamic model of a mobile manipulator, comprising a four-wheels vehicle and a two-DOF robotic manipulator, is a differentially flat one. The differential flatness property of the model confirmed that this could be transformed into an inputoutput linearized form and that it could be also written in an equivalent linear canonical (Brunovsky) form. For the latter representation of the system's dynamics the solution of both the control and state-estimation problem has become possible. Actually, it has been shown that one can stabilize the mobile manipulator by applying a pole placement technique on its linearized equivalent model.

Moreover, to perform state estimation the Derivativefree nonlinear Kalman Filter has been introduced. This consists of the recursion of the standard Kalman Filter applied on the linearized equivalent model of the mobile manipulator and of an inverse transformation based on differential flatness theory which allows for computing estimates of the state variables of the initial nonlinear model of the robot. Additionally, to estimate and compensate for model uncertainty and external disturbances affecting the mobile manipulator, the aforementioned Kalman Filter has been redesigned as a disturbance observer. To this end, the state vector of the mobile manipulator has been extended by including as additional state variables the disturbance inputs and their time-derivatives. By obtaining accurate estimates of such perturbation terms their compensation has become also possible.

\section{References}

Abeygunawardhana P.K.W. and Murakami T. (2010). Vibration suppression of two-wheel mobile manipulator using resonance-ratio-control-based null-space control, IEEE Transactions on Industrial Electronics, vol. 57, no. 12, pp. 4137-4146.

Andaluz V.H., Roberi F., Salinas L., Toibeiro J.M., and Carelli R. (2015). Passivity-based visual feedback control with dynamic compensation of mobile manipulators: Stability and $L_{2}$-gain performance analysis, Robotics and Autonomous Systems, Elsevier, vol.66, pp.64-74.

Basseville M. and Nikiforov I. (1993). Detection of Abrupt Changes: Theory and Applications, PrenticeHall.

Boyle B., Fourquet J.Y., and Renaud M. (2001). Manipulability of wheeled mobile manipulators: Application to motion generation, Intl. Journal of Robotic Research, J. Wiley, vol. 22, no 7-8, pp. 565-581.

Dai G.B. and Liu Y.C. (2017). Distributed coordination and cooperation control for networked mobile manipulators, IEEE Transactions on Industrial Electronics, vol. 64, no. 6, pp. 5065-5074.

Fliess M. and Mounier H. (1999). Tracking control and $\pi$-freeness of infinite dimensional linear systems, In: G. Picci and D.S. Gilliam Eds. Dynamical Systems, Control, Coding and Computer Vision, vol. 258, pp. 41-68, Birkhaüser.

Galicki M. (2016). Real-time constrained trajectory generation of mobile manipulators, Robotics and $\mathrm{Au}$ tonomous Systems, Elsevier, vol. 78, pp.49-62.

Kocemarek M., Domski W., and Mazur A. (2017). Position-force control of mobile manipulator - nonadaptive and adaptive case, Archives of Control Sciences, DeGruyter, vol. 27, no. 4, pp. 487-503.

Koraye M.H. amd Nekao S.R. (2016). The SDRE control of mobile-base cooperative manipulators: Collision-free path planning and moving obstable avoidance, Robotics and Autonomous Systems, Elsevier, vol. 88, pp. 86-105.

Lévine, J. (2011). On necessary and sufficient conditions for differential flatness, Applicable Algebra in Engineering, Communications and Computing, Springer, vol. 22, no. 1, pp. 47-90,

Li Z., Tan P.Y., Ge S.S., Adams M., and Wijesoma W.S. (2009). Robust adaptive control of cooperative mobile manipulators with relative motion, IEEE Transactions on Systems, Man and Cybernetics — Part B: Cybernetics, vol. 37, no.1, pp. 103-116.

Li Z., Tan P.Y., Ge S.S., Adams M., and Wijesoma W.S. (2008). Adaptive robust output-feedback motion/force control of electrically driven nonholonomic mobile manipulators, IEEE Transactions on Control Systems Technology, vol. 16, no. 6, pp. 1308-1315.

Li Z., Tan P.Y., Ge S.S., Adams M., and Wijesoma W.S. (2008). Robust adaptive control of uncertain force/motion constrained nonholonomic mobile manipulators, Automatica, Elsevier, vol. 44, pp. 776-784.

Li Z., Ge S.S., and Ming A. (2016). Adaptive robust motion/force control of holonomic-constrained nonholonomic mobile manipulators, IEEE Transactions on Systems, Man and Cybernetics: Part B-Cybernetics, vol. 37, no. 3, pp. 607-616.

Li, Z., Yang, Y., and Li, J. (2010). Adaptive motion/force control of mobile underactuated manipulators with dynamics uncertainty by dynamic coupling and output feedback, IEEE Transactions on Control Systems Technology, vol. 18, no. 5, pp. 1068-1079.

Lin, S. and Goldenberg, A. (2002). Robust damping control of mobile manipulators, IEEE Transactions on Systems, Man and Cybernetics: Part B - Cybernetics, vol. 32, no. 1, pp. 126-132.

Liu, Y. and Liu, G. (2009). Modelling of tracked mobile manipulators with consideration of truck-terrain 
and vehicle-manipulator interactions, Robotics and $\mathrm{Au}$ tonomous Systems, Elsevier, vol. 57, pp. 1065-1074.

Mai, T. and Wang, Y. (2014). Adaptive Force/Motion control system based on recurrent fuzzy wavelet CMAC neural networks for condenser cleaning crawler-type mobile manipulator robot, IEEE Transactions on Control Systems Technology, vol. 22, no. 5, pp. 1973-1982.

Monzur, A. and Kulawik, M. (2006). Adaptive trajectory tracking for doubly-fed nonholonomic mobile manipulator - 3 pendulum on mobile platform of (2.0) Class, 8th IFAC Symposium on Robot Control, Syroco 2006, Bologna, Italy.

Morales, R., Sira-Ramirez, H., and Somolinos, J.A. (2014). Robust control of underactuated wheeled mobile manipulators using GPI disturbance-observers, Multibody System Dynamics, Springer, vol. 32, no. 4, pp. 511-533.

Najjaran, H. and Goldenberg, A. (2007). Real-time motion planning of an autonomous mobile manipulator using fuzzy adaptive Kalman Filter, Robotics and Autonomous Systems, Elsevier, vol. 55, pp. 96-106.

Park, B.S., Park, J.B., and Choi, Y.H. (2018). Adaptive observer-based trajectory tracking control of nonholonomic mobile robots, International Journal of Control, Automation and Systems, vol. 9, no. 3, pp 534-541.

Rigatos, G.G. and Tzafestas, S.G. (2007). Extended Kalman filtering for fuzzy modelling and multi-sensor fusion, Mathematical and Computer Modelling of Dynamical Systems, Taylor \& Francis, vol. 13, pp. 251266.

Rigatos, G. and Zhang, Q. (2009). Fuzzy model validation using the local statistical approach, Fuzzy Sets and Systems, Elsevier, 60 (7), pp. 882-904.

Rigatos, G. (2011). Modelling and Control for In- telligent Industrial Systems: Adaptive Algorithms in Robotcs and Industrial Engineering, Springer. Rigatos, G. (2015). Nonlinear Control and Filtering Using Differential Flatness Approaches: Applications to Electromechanicsl Systems, Springer.

Rigatos, G. and Busawon, K. (2018). Robotic Manipulators and Vehicles: Control, Estimation and Filtering, Springer.

Sira-Ramirez, H. and Agrawal, S. (2004). Differentially Flat Systems, Marcel Dekker, New York.

Souzanchi, M., Arab, A., Akbarzadeh, M.R., and Fetch M.M. (2017). Rovust impendance control of uncertain mobile manipulators using time-delay compensation, IEEE Transactions on Control Systems Technology.

Tang, C.P., Miller, P.T., Kravi, V.N., Rya, J.C., and Agrawal, S.K. (2011). Differential-flatness-based planning and control of a wheeled mobile manipulator theory and experiments, IEEE Transactions on Mechatronics, vol. 16, no. 5, pp. 768-773.

Villagra, J., d'Andrea-Novel, B., Mounier, H., and Pengov, M. (2007). Flatness-based vehicle steering control strategy with SDRE feedback gains tuned via a sensitivity approach, IEEE Transactions on Control Systems Technology, vol. 15, pp. 554-565.

Wu, X., Wang, Y., and Dong, X. (2014). Robust adaptive sliding-mode control of condense-cleaning mobile manipulator using fuzzy wavelet neural network, Fuzzy Sets and Systems, Elsevier, vol. 235, pp. 62-82.

Xu, D., Zhao, D., Yi, J., and Tan, X. (2009). Trajectory tracking control of omnidirectional wheeled mobile manipulators: Robust neural network-based slidingmode approach, IEEE Transactions on Systems, Man and Cybernetics: Part B-Cybernetics, vol. 39, no. 3, pp. 788-799. 